\title{
Functional brain fluorescence plurimetry in rat by implantable concatenated CMOS imaging system
}

\author{
Takuma Kobayashi $^{\mathrm{a}, *}$, Hiroyuki Masuda ${ }^{\mathrm{b}}$, Chikara Kitsumoto ${ }^{\mathrm{b}}$, Makito Haruta ${ }^{\mathrm{b}}$, \\ Mayumi Motoyama ${ }^{\mathrm{b}}$, Yasumi Ohta ${ }^{\mathrm{b}}$, Toshihiko Noda ${ }^{\mathrm{b}}$, Kiyotaka Sasagawa ${ }^{\mathrm{b}}$, Takashi \\ Tokuda $^{\mathrm{b}}$, Sadao Shiosaka ${ }^{\mathrm{c}}$, and Jun Ohta ${ }^{\mathrm{b}}$
}
${ }^{a}$ Division of Molecular Brain Science, Research Institute of Traditional Asian Medicine, Kinki University, Osaka-Sayama, Osaka 589-8511, Japan
${ }^{\mathrm{b}}$ Graduate School of Materials Science, Nara Institute of Science and Technology, Ikoma, Nara 630-0192, Japan
${ }^{c}$ Graduate School of Biological Sciences, Nara Institute of Science and Technology, Ikoma, Nara 630-0192, Japan

*Corresponding author. Tel.: +81 74372 6054; fax: +81 743726052 .

E-mail address: ta-kobay@med.kindai.ac.jp, yaemugura@gmail.com (T. Kobayashi).

Keywords: CMOS image sensor, implantable microsensor, functional brain imaging, fluorescence potentiometry, voltage-sensitive dye, rat somatosensory cortex

\begin{abstract}
Measurement of brain activity in multiple areas simultaneously by minimally invasive methods contributes to the study of neuroscience and development of brain machine interfaces. However, this requires compact wearable instruments that do not inhibit natural movements. Application of optical potentiometry with voltage-sensitive fluorescent dye using an implantable image sensor is also useful. However, the increasing number of leads required for the multiple wired sensors to measure larger domains inhibits natural behavior. For imaging broad areas by numerous sensors without excessive wiring, a web-like sensor that can wrap the brain was developed. Kaleidoscopic potentiometry is possible using the imaging system with concatenated sensors by changing the alignment of the sensors. This paper describes organization of the system, evaluation of the system by a fluorescence imaging, and finally, functional brain fluorescence plurimetry by the sensor. The recorded data in rat somatosensory cortex using the developed multiple-area imaging system compared well with electrophysiology results.
\end{abstract}




\section{Introduction}

In recent years, several kinds of instrument for functional brain measurements have been developed to reveal neural activities and provide insights into animal behavior in neuroscience. For example, fMRI (functional magnetic resonance imaging) enables non-invasive functional brain analysis (Ogawa et al., 1990), fNIRS (functional near-infrared spectroscopy) and optical topography do not obstruct the action of the subject by using the wearable probes (Kato et al., 1993; Hoshi and Tamura, 1993; Maki et al., 1995). PET (positron emission tomography), EEG (electroencephalography), and a small microscope of the head installation type have been developed for freely moving animals (Sossi and Ruth, 2005; Schulz et al., 2011; Ishida et al., 1993; Fan et al., 2011, Helmchen et al., 2001; Park et al., 2011). Each technology has, however, both advantages and disadvantages.

Ideal functional brain measurements require a minimally invasive method and a measurement area that is as wide as possible. It is also preferable that the measured subject can move freely. Optical imaging is a useful and powerful tool for such measurements. It enables wide-ranging analyses with high spatiotemporal resolution without destroying tissue. In particular, voltage-sensitive dye imaging, which visualizes a variation in a membrane potential as a change in fluorescence intensity, can be used for real-time analysis of the activity of individual neurons directly (Tasaki et al., 1968; Cohen et al., 1968, 1978; Davila et al., 1973; Salzberg et al., 1973; Grinvald et al., 1986; Grinvald and Hildesheim, 2004). Implantable complementary-metal-oxide-semiconductor (CMOS) image sensors enable less-invasive fluorescence imaging in living tissue ( $\mathrm{Ng}$, et al., 2006; 2008; Tamura et al., 2008; Tagawa et al., 2010; Ohta et al., 2009; 2011). A previous study demonstrated a fluorescence imaging system incorporating a tiny implantable sensor for a freely moving animal. The system enabled potentiometry in primary cultured neurons and in the brain (Kobayashi et al., 2010, 2011a, 2012). These compact instruments for functional brain measurements with a freely moving animal can provide insight into the natural behavior of animals. However, in these conventional imaging systems, the accumulation of leads and wearing transit boards attached to the sensors inhibits the animal behavior as the number of sensors increases (Kobayashi et al., 2011b, 2012). To solve this problem, we concatenated the sensors and show that the new imaging system actually enables potentiometric imaging of multiple areas in the brain. To the best of our 
knowledge, this is the first report in which a large number of CMOS image sensors were simultaneously applied to the living brain. The plurimetry in larger domains as a type of whole-brain imaging in freely moving animals could reveal various connections between distinct brain regions.

\section{Material and Methods}

2.1 Design and development of fluorescence multiple-area imaging system.

The accumulation of leads accompanying multiple sensors attached to an animal inhibits free movement (Fig. 1 A). An imaging system designed with a CMOS chip assures that multiple sensors are simultaneously driven by fewer leads. The linearity of the trigger signals for the start sequence and the consecutive imaging data contributes to retrench total lines and constitutions without the addition of another line (Fig. 1 B, see Sasagawa et al., 2011 for details). As the output signal from each sensor is sent in turn, it does not interfere each other on the shared one line. The CMOS imaging sensors and light-emitting diodes (LEDs) are arranged with wire-bonding on a flexible polyimide substrate, which can be applied atraumatically. (Fig. 1 C). The excitation light of the LED (470-490 nm, Epistar Corp, Taiwan) elicits fluorescence from neural cells stained with a fluorescent dye. The fluorescence passes through a red absorption filter (high-pass liquid photoresist filter; >600 nm, FUJIFILM Corp., Japan) that is superimposed over the pixel array, while the excitation light is blocked. The sensor was covered by a waterproof resin. The imaging sensor was fabricated using a $0.35 \mu \mathrm{m}$ 2-poly 4-metal standard CMOS process from Austria Micro Systems, based on a 3 -transistor active pixel sensor (Fig. 1 D). The design was based on previous work (Tagawa et al., 2009; Sasagawa et al., 2011). The sensor is $7.5 \mu \mathrm{m} \times 7.5 \mu \mathrm{m}$ and the number of pixels is $60 \times 60$, which corresponds to an imaging area of $450 \mu \mathrm{m} \times 450 \mu \mathrm{m}$. The analog output data stream from the sensor was transferred by a transit board, pre-amplified and converted to 12-bit digital data by a control board, and the digital data were processed by custom-developed software. One computer was used to control the multiple sensors and also record and store the image data.

2.2 Fluorescence measurement of fluorescent beads and liquid.

The fabricated sensor was placed in a dish and immersed in physiological saline. 
Fluorescent beads (F21012; Excitation (Ex)/Emission (Em) wavelength $=559 / 580 \mathrm{~nm}$, Life Technologies Corp., Japan), and a resorufin (7-hydroxy-3H-phenoxazin-3-one, $\mathrm{Ex} / \mathrm{Em}=570 / 585 \mathrm{~nm}$, Tokyo Chemical Industry Co., Ltd., Japan) as a fluorescent liquid were used. For fluorescence imaging with the sensor, the beads were scattered onto the sensor (Fig. $2 \mathrm{~A}$ ), and $100 \mu \mathrm{M}$ resorufin/50\% glycerol solution was dropped using a micropipette (Fig. 2 B, white cross shows the drop point). In both cases, the excitation light reflected from the surface of the salt solution was subtracted as a baseline with imaging software, and artifact signals, such as an imperceptible ripple, were not detected by the sensor.

\subsection{Surgery and recording.}

All animal procedures conformed to the animal care and experimentation guidelines of Nara Institute of Science and Technology. For in vivo experiments, Sprague-Dawley rats (300-500 g) (SLC Inc., Japan) were anaesthetized with urethane (1.5 g/kg) and held with a stereotaxic instrument (Narishige Corp, Japan). Fluorescence imaging with the sensor and electrophysiological recording were performed as described previously (Kobayashi et al., 2012). Briefly, after craniotomy, the rat cortex was stained with voltage-sensitive dye, RH795 (Life Technologies Corp.). Artificial cerebrospinal fluid (ACSF) containing (in mM): $124 \mathrm{NaCl}, 3.0 \mathrm{KCl}, 1.25 \mathrm{KH}_{2} \mathrm{PO}_{4}, 10$ D-Glucose, 1.0 $\mathrm{MgSO}_{4}, 26 \mathrm{NaHCO}_{3}, 2.0 \mathrm{CaCl}_{2}, \mathrm{pH}$ 7.4, was used to keep the cortical surface moist. After confirming the appropriate fluorescence signal by stereo-microscope, the device was applied to the cortex (Fig. 3 A). Generally, animal's head is strictly fixed during fluorescence-imaging microscopy, and use of an artificial heart-lung apparatus and an anti-vibration table to avoid a noise is preferred. Fortunately, the applied CMOS sensor moves in conjunction with the breathing and heartbeat of the host rat. This makes measurements simple and easy, which are advantages over other imaging modalities.

\section{Results and Discussion}

3.1. Utility test of fabricated fluorescence multiple-area imaging system.

To verify whether the sensor was waterproof and capable of taking fluorescence images at cellular resolution, images of $15-\mu \mathrm{m}$-diameter fluorescent beads in physiological saline were acquired using either the sensor or a stereo-microscope (Fig. 2 
A). Comparing the left fluorescence images captured by the microscope with the right pseudo-color images of data obtained by the sensor, the sensor detects the fluorescence and can discriminate individual beads. Dipping the device into the salt solution did not disrupt the signal or damage the device.

It was subsequently verified that dynamic fluorescence signals that change spatiotemporally could be detected by each sensor. The sensor was immersed in saline, and then, resorufin was applied gently under the surface of the saline using a micropipette, without disturbing the surface of the saline (Fig. 2 B). Pseudo-colored time-lapse images captured by the sensor are shown in Fig. 2 C. Red squares and broken circles in Fig. 2 B show a primary wave arrival of the resorufin to each sensor. Judging from the time of the primary wave arrivals, the resorufin spreads and passes at 1.2-5.1 $\mu \mathrm{m} / \mathrm{ms}$ over each sensor, and the initial velocity of the resorufin is $8.9 \mu \mathrm{m} / \mathrm{ms}$ ( $\mathrm{y}=$ $\left.0.0089 \mathrm{e}^{-0.28 \mathrm{x}}, \mathrm{R}^{2}=0.978\right)$. Averaging the signal, an effective best imaging speed of the sensor with a low noise and a cellular resolution necessary for imaging a living brain with voltage-sensitive dye is $\sim 38.0 \mathrm{~ms} /$ frame at $120 \times 268$ pixels (Kobayashi, et al., 2012). By improving the imaging software to include signal rectification processing and developing a new control board, the sensor could be driven at a maximum speed of 14.0 $\mathrm{ms} /$ frame with low noise for 8 sensors of $60 \times 60$ pixels. The distance between sensors 1 and 8 is $\sim 7 \mathrm{~mm}$. This indicates that the imaging system can identify a fluorescent substance at a maximum speed of $\sim 500 \mu \mathrm{m} / \mathrm{ms}$. The imaging system can be driven at a higher speed by reducing the number of drive sensors and limiting the number of pixels in the image. For instance, the latency difference of $\sim 9 \mathrm{~ms}$ between $\mathrm{S} 1$ and M1 activity is consistent with a pyramidal neuron axonal conduction velocity of $\sim 450 \mu \mathrm{m} / \mathrm{ms}$ in mouse (Meeks and Mennerick, 2007; Ferezou et al., 2007). Even if there is a natural delay in the propagation by other indirect pathways, the sensor is able to image all events. Although the diffusion speed of resorufin might be too slow for the evaluation, the fundamental properties of the device for fluorescence imaging were verified. Each sensor was able to discriminate a dynamic temporal change in the fluorescence intensity, and visualize the spatial separation of the fluorescence. For detailed evaluation of the imaging signal, concatenated signal transferals and improvement between two sensors in this system have been described in Sasagawa et al., 2011.

3.2. Functional fluorescence brain imaging of multiple areas on the rat somatosensory and parietal association cortices.

Brain imaging with voltage-sensitive dye using the sensor was performed in a rat 
(Fig. 3 A). After craniotomy and staining of the cortex with fluorescent dye, the device was implanted at the position shown in Fig. $3 \mathrm{~B}$ (the rat brain map according to Paxinos and Watson, 1998; Krubitzer et al., 2011). Images of the fluorescence data obtained by each sensor are shown as grayscale images in the left row in Fig. $3 \mathrm{C}$. The sensor data represent the membrane potential levels as the fluorescence intensity in cortical neural cells. When the trunk of the rat was pinched, changes in fluorescence, shown as pseudo-colored time-lapse images, were seen in the somatosensory and posterior parietal association cortices (Reep et al., 1994). The spatiotemporal changes were analyzed in detail (Fig. 3 D, E). The ratio of change in the fluorescence intensity indicates an inverted membrane potential, considering the RH795 specifications. As the standard value was set low to emphasize the positive changes that are relatively weak compared to the negative changes, the signal detected by sensor 1 seems to be earlier than the one detected by sensor 3 (Fig. 3 C). However, the signal detected by sensor 3 seems to be earlier than the one detected by sensor 1 rather than both are approximately simultaneous judging from the actual changes of the fluorescence intensity (Fig. $3 \mathrm{E}$; especially comparison between ROI 5, 6 and ROI 8, 9, 11). These results suggest that the neural activities propagate from S1Tr to PPC. These imaging data at least show that burst firing was elicited according to the sensory stimulation. Actually, multi-unit activity by electrophysiological measurement of local field potential (LFP) was detected in S1Tr and PPC, but not in S1 near sensor 8 (Fig. $3 \mathrm{~F}$ i-iii). The changes in LFP were converted to pseudo colors and averaged. Although some neural reactions may be slightly attenuated with short intervals of stimulation (Fig. $3 \mathrm{Fi}$ ), the pseudo-colored regions reveal that positive potential changes dominantly occurred at PPC depending on the stimulation, while negative potential changes dominantly occurred at S1Tr (Fig. $3 \mathrm{~F}$ i, ii). These results show a trend similar to the results of the optical imaging. This phenomenon would be conveniently explained as a burst from neurons, such as pyramidal neurons at S1Tr that occurred intensely over a wide area (Fig. 3 D c-3, 3 E c-3). Sequentially, the activated signals were propagated and evoked neurons, such as GABAergic neurons, at PPC, which dominantly inhibited surrounding neurons (Fig. 3 D c-1, 3 E c-1). These serial sideling responses may indicate close signal transduction from S1 to PPC associated with higher brain dysfunction, or the parallel progression concerning the cortex-basal ganglia loop including thalamus transmitting the inhibitory action in PPC. While it is difficult to discuss the time lag of the propagation using only the results from the current experimental system, these results may be hinting at an important biological finding. Future studies, including surgical lesion of the transmission pathway, multineuron patch-clamp recordings, and optogenetic approach 
for specific neuronal responses, are needed. The developed imaging system does enable multipoint potentiometric analysis in a broad area of the brain. A mathematical prediction method, such as the spike sorting that is performed in electrocorticography, would help discriminate the individual neural activity of deep brain regions. Ideally, individual neuronal activity should be visualized in the whole brain. On the other hand, observance of the orchestrated neural activities of a group of neurons is important to interpret meaningful activity of the brain. For whole-brain imaging, such as measurements of the superficial cortex activity, the imaging system can reveal spatiotemporal activity between distinct brain areas concerning animal behavior and emotion.

\section{Conclusions}

For imaging broad areas of the brain, a fluorescence imaging system incorporating several implantable CMOS image sensors was developed. The system was capable of both in vitro and in vivo fluorescence multi-imaging. Using multiple sensors, the system can monitor the fluctuation of the membrane potential in cortical neural cells.

\section{Acknowledgement}

This work was supported by the Core Research for Evolutional Science and Technology (CREST) from Japan Science and Technology Agency (JST), and the Grant-in-Aid for Scientific Research (23246068) and the Young Scientists (24700411) from the Japan Society for the Promotion of Science (JSPS).

\section{Figure captions}

Fig. 1

Schematic diagram of micro CMOS image sensor for imaging of multiple brain areas. (A, B) One CMOS sensor requires four leads: the supply voltage (Vdd), data output (Vout), clock signal (Clk) and ground (Gnd) (A). Conventionally, each image sensor is driven individually by a separate PCI connector, control board, and transit board. As the number of sensors increases, the overall imaging system becomes unreasonably large. 
To simplify the system, multiple sensors are linked together, sharing lines, boards and connectors (B). A fifth line provides a start signal to the sensors, and all the sensors act in sequence (red line in B). (C) The fluorescence imaging method using the sensor. A blue excitation light from a light-emitting diode(LED) excites a neuron stained with a fluorescent dye (1). The red fluorescence emitted from the neuron passes through an absorption filter covering the sensor (2); the blue exciting light from the LED is blocked (3). The sensor and LED are arranged on a flexible polyimide substrate, and multiple pairs are assembled into a single device. (D) The multiple-area imaging system is shown. A control board connects the imaging device through a transit board, and multiple sensors are controlled by one PC.

Fig. 2

(A) The imaging device was immersed in physiological saline, and fluorescent beads were scattered on the sensor. The fluorescent beads, $15 \mu \mathrm{m}$ in diameter, were exposed to light from the LEDs of the device. The fluorescence image is then detected by the sensor or a microscope set on the device. (the numbers 1-8 indicate the sensor positions in $\mathrm{B}$, pseudo-color image $=$ data obtained by the sensor, white broken line $=$ pixel area of the sensor) (B-D) When a red fluorescent liquid is dropped near the device immersed in physiological saline (white cross in B), the liquid immediately spreads over the device. The spreading process was captured using the sensor and the LEDs. The movie was recorded at $15.6 \mathrm{~ms} /$ frame, and still images are shown as pseudo-color time-lapse images $(\mathrm{C})$. The red-circled frame represents the moment when the first fluorescent wave reached each sensor. The temporal changes in the fluorescence intensity measured by each sensor are shown in (D).

Fig. 3

Fluorescence imaging of the rat brain stained with voltage-sensitive dye was performed using the implantable CMOS image sensor. (A) Image showing the experiment. The device was implanted on the somatosensory cortex of the rat after craniotomy. The number 1 sensor located to $+4.5 \mathrm{~mm}$ lambda and $+3.5 \mathrm{~mm}$ lateral. (B) A schematic image of the brain map $(\mathrm{V} 1 / 2=$ primary/secondary visual cortex, $\mathrm{PPC}=$ posterior parietal cortex, $\mathrm{S} 1 / 2=$ primary/secondary somatosensory cortex, $\mathrm{Tr}=$ trunk region, $\mathrm{BF}$ = barrel field, $\mathrm{M} 1 / 2=$ primary/secondary motor cortex). The broken square and numbers indicate the position of each sensor of the imaging device. Blue asterisks in (B) and (C) adjacent to sensor 1 mean an identical orientation, which is the same for the other sensor. For electrophysiological measurements, the electrodes were inserted near 
sensors 1, 3 and 8 (white arrowhead in B; i-iii), and the reference electrode was inserted on the opposite side (black arrowhead in A). (C) A movie was captured using the implanted sensor, and still images are shown as pseudo-color time-lapse images. Nociceptive (pinch) stimulation in the rat trunk causes transient changes in the fluorescence (red bar = stimulation). (D) Pseudo-color images derived from one frame captured by sensors 1 and 3 in (C). The numbers indicate a region of interest (ROI) of (E) correspondingly. (E) shows the temporal change in the fluorescence intensity in each ROI. (red bar = stimulation) (F) The local field potentials at (i), (ii) and (iii) in (B) are shown. The upper pseudo-color bars show the potential changes from $-30 \mu \mathrm{V}$ to +30 $\mu \mathrm{V}$, with a gradient of blue-yellow-red. The lower bars show the average of the upper bars. $($ red bar $=$ stimulation $)$

\section{References}

Cohen, L.B., Keynes, R.D., Hille, B., 1968. Light scattering and birefringence changes during nerve activity. Nature 218(5140), 438-441.

Cohen, L.B., Salzberg, B.M., Grinvald, A., 1978. Optical methods for monitoring neuron activity. Annu Rev Neurosci 1, 171-182.

Davila, H.V., Salzberg, B.M., Cohen, L.B., Waggoner, A.S., 1973. A large change in axon fluorescence that provides a promising method for measuring membrane potential. Nat New Biol 241(109), 159-160.

Fan, D., Rich, D., Holtzman, T., Ruther, P., Dalley, J.W., Lopez, A., Rossi, M.A., Barter, J.W., Salas-Meza, D., Herwik, S., Holzhammer, T., Morizio, J., Yin, H.H., 2011. A wireless multi-channel recording system for freely behaving mice and rats. PLoS One 6(7), e22033.

Ferezou, I., Haiss, F., Gentet, L.J., Aronoff, R., Weber, B., Petersen, C.C., 2007. Spatiotemporal dynamics of cortical sensorimotor integration in behaving mice. Neuron 56(5), 907-923.

Paxinos, G., Watson, C., 1982. The Rat Brain, Sixth edition 2007 ed. Academic Press is an imprint of Elsevier, London, UK.

Grinvald, A., Hildesheim, R., 2004. VSDI: a new era in functional imaging of cortical dynamics. Nat Rev Neurosci 5(11), 874-885.

Grinvald, A., Lieke, E., Frostig, R.D., Gilbert, C.D., Wiesel, T.N., 1986. Functional architecture of cortex revealed by optical imaging of intrinsic signals. Nature 324(6095), 361-364. 
Helmchen, F., Fee, M.S., Tank, D.W., Denk, W., 2001. A miniature head-mounted two-photon microscope. high-resolution brain imaging in freely moving animals. Neuron 31(6), 903-912.

Hoshi, Y., Tamura, M., 1993. Detection of dynamic changes in cerebral oxygenation coupled to neuronal function during mental work in man. Neurosci Lett 150(1), 5-8.

Ishida, N., Kasamo, K., Nakamoto, Y., Suzuki, J., 1993. Epileptic seizure of El mouse initiates at the parietal cortex: depth EEG observation in freely moving condition using buffer amplifier. Brain Res 608(1), 52-57.

Kato, T., Kamei, A., Takashima, S., Ozaki, T., 1993. Human visual cortical function during photic stimulation monitoring by means of near-infrared spectroscopy. J Cereb Blood Flow Metab 13(3), 516-520.

Kobayashi, T., Motoyama, M., Masuda, H., Ohta, Y., Haruta, M., Noda, T., Sasagawa, K., Tokuda, T., Tamura, H., Ishikawa, Y., Shiosaka, S., Ohta, J., 2012. Novel implantable imaging system for enabling simultaneous multiplanar and multipoint analysis for fluorescence potentiometry in the visual cortex. Biosens Bioelectron 38(1), 321-330.

Kobayashi, T., Noda, T., Sasagawa, K., Tokuda, T., Shiosaka, S., Ohta, J., 2011b. In vivo imaging of mouse with CMOS image sensor. Optronics 30(10), 99-104. (ISSN: 02869659)

Kobayashi, T., Tagawa, A., Noda, T., Sasagawa, K., Tokuda, T., Hatanaka, Y., Tamura, H., Ishikawa, Y., Shiosaka, S., Ohta, J., 2010. Potentiometric dye imaging for pheochromocytoma and cortical neurons with a novel measurement system using an integrated complementary metal-oxide-semiconductor imaging device. Jpn J Appl Phys 49(11), 117001.

Kobayashi, T., Tamura, H., Hatanaka, H., Motoyama, M., Noda, T., Sasagawa, K., Tokuda, T., Ishikawa, Y., Shiosaka, S., Ohta, J., 2011a. Functional neuroimaging by using an implantable CMOS multimodal device in a freely-moving mouse. Proc IEEE BioCAS(10-12 Nov. 2011), 110-113.

Krubitzer, L., Campi, K.L., Cooke, D.F., 2011. All rodents are not the same: a modern synthesis of cortical organization. Brain Behav Evol 78(1), 51-93.

Maki, A., Yamashita, Y., Ito, Y., Watanabe, E., Mayanagi, Y., Koizumi, H., 1995. Spatial and temporal analysis of human motor activity using noninvasive NIR topography. Med Phys 22(12), 1997-2005.

Meeks, J.P., Mennerick, S., 2007. Action potential initiation and propagation in CA3 pyramidal axons. J Neurophysiol 97(5), 3460-3472.

Ng, D.C., Tamura, H., Mizuno, T., Tokuda, T., Nunoshita, M., Ishikawa, Y., Shiosaka, 
S., Ohta, J., 2008. An implantable and fully integrated complementary metal-oxide semiconductor device for in vivo neural imaging and electrical interfacing with the mouse hippocampus. Sensors \& Actuators A 145-146, 176-186.

Ng, D.C., Tamura, H., Tokuda, T., Yamamoto, A., Matsuo, M., Nunoshita, M., Ishikawa, Y., Shiosaka, S., Ohta, J., 2006. Real time in vivo imaging and measurement of serine protease activity in the mouse hippocampus using a dedicated complementary metal-oxide semiconductor imaging device. J Neurosci Methods 156(1-2), 23-30.

Ogawa, S., Lee, T.M., Kay, A.R., Tank, D.W., 1990. Brain magnetic resonance imaging with contrast dependent on blood oxygenation. Proc Natl Acad Sci U S A 87(24), 9868-9872.

Ohta, J., Kobayashi, T., Noda, T., Sasagawa, K., Tokuda, T., 2011. CMOS imaging devices for biomedical applications. IEICE Transactions on Communications E94.B(9), 2454-2460.

Ohta, J., Tokuda, T., Sasagawa, K., Noda, T., 2009. Implantable CMOS biomedical devices. Sensors (Basel) 9(11), 9073-9093.

Park, J.H., Platisa, J., Verhagen, J.V., Gautam, S.H., Osman, A., Kim, D., Pieribone, V.A., Culurciello, E., 2011. Head-mountable high speed camera for optical neural recording. J Neurosci Methods 201(2), 290-295.

Reep, R.L., Chandler, H.C., King, V., Corwin, J.V., 1994. Rat posterior parietal cortex: topography of corticocortical and thalamic connections. Exp Brain Res 100(1), 67-84.

Salzberg, B.M., Davila, H.V., Cohen, L.B., 1973. Optical recording of impulses in individual neurones of an invertebrate central nervous system. Nature 246(5434), 508-509.

Sasagawa, K., Masuda, H., Tagawa, A., Kobayashi, K., Noda, T., Tokuda, T., Ohta, J., 2011. Micro CMOS image sensor for multi-area imaging. IFIP/IEEE International Conference on Very Large Scale Integration (VLSI-SoC2011), Hong Kong, China.

Schulz, D., Southekal, S., Junnarkar, S.S., Pratte, J.F., Purschke, M.L., Stoll, S.P., Ravindranath, B., Maramraju, S.H., Krishnamoorthy, S., Henn, F.A., O'Connor, P., Woody, C.L., Schlyer, D.J., Vaska, P., 2011. Simultaneous assessment of rodent behavior and neurochemistry using a miniature positron emission tomograph. Nat Methods 8(4), 347-352.

Sossi, V., Ruth, T.J., 2005. Micropet imaging: in vivo biochemistry in small animals. J Neural Transm 112(3), 319-330.

Tagawa, A., Higuchi, A., Sugiyama, T., Sasagawa, K., Tokuda, T., Tamura, H., 
Hatanaka, Y., Ishikawa, Y., Shiosaka, S., Ohta, J., 2009. Development of complementary metal oxide semiconductor imaging devices for detecting green fluorescent protein in the deep of a freely moving mouse. Jpn J Appl Phys 48, $04 \mathrm{C} 195$.

Tagawa, A., Minami, H., Mitani, M., Noda, T., Sasagawa, K., Tokuda, T., Tamura, H., Hatanaka, H., Ishikawa, Y., Shiosaka, S., Ohta, J., 2010. Multimodal complementary metal-oxide-semiconductor sensor device for imaging of fluorescence and electrical potential in deep brain of mouse. Jpn J Appl Phys 49(1), $01 \mathrm{AG} 02$.

Tamura, H., Ng, D.C., Tokuda, T., Naoki, H., Nakagawa, T., Mizuno, T., Hatanaka, Y., Ishikawa, Y., Ohta, J., Shiosaka, S., 2008. One-chip sensing device (biomedical photonic LSI) enabled to assess hippocampal steep and gradual up-regulated proteolytic activities. J Neurosci Methods 173(1), 114-120.

Tasaki, I., Watanabe, A., Sandlin, R., Carnay, L., 1968. Changes in fluorescence, turbidity, and birefringence associated with nerve excitation. Proc Natl Acad Sci U S A 61(3), 883-888. 


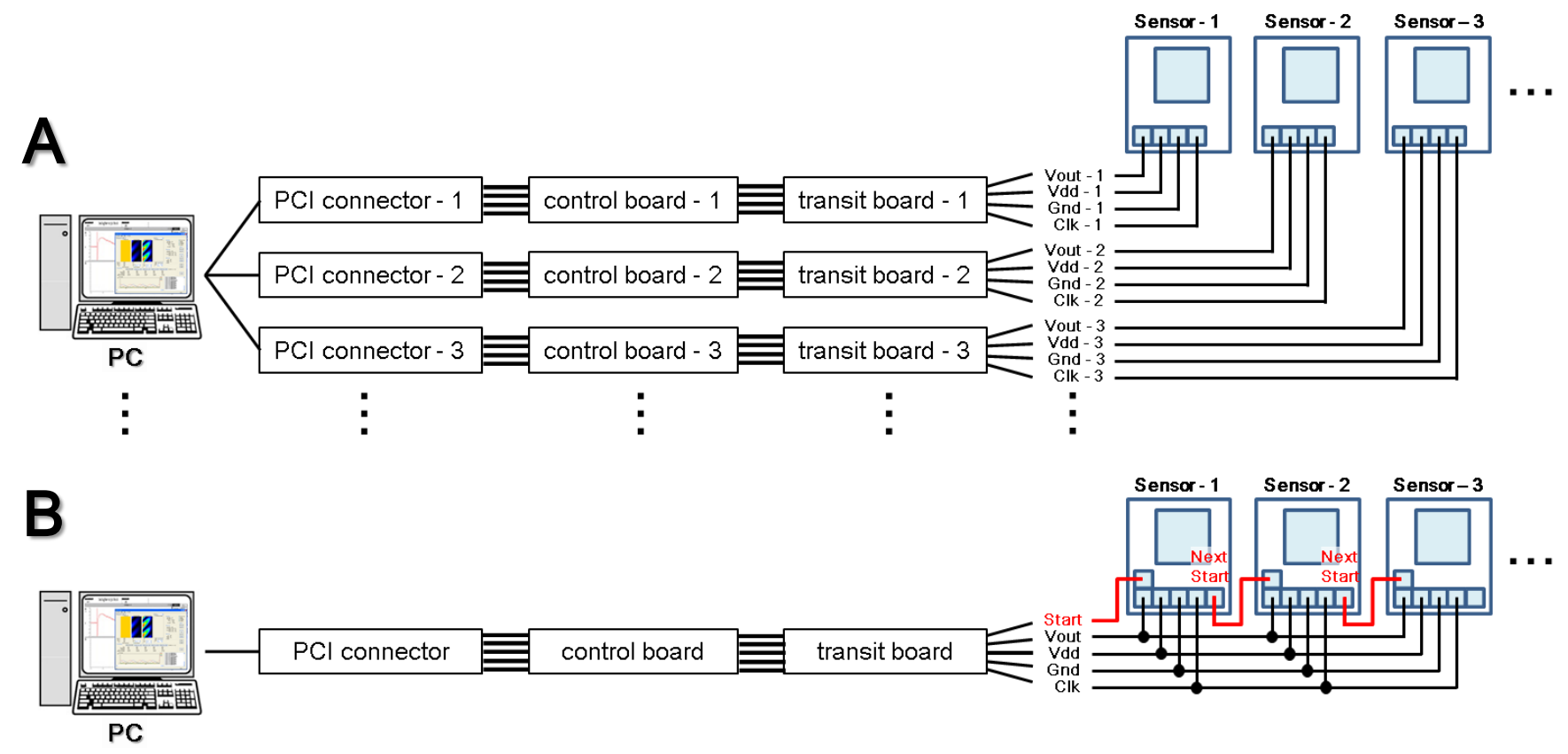

C

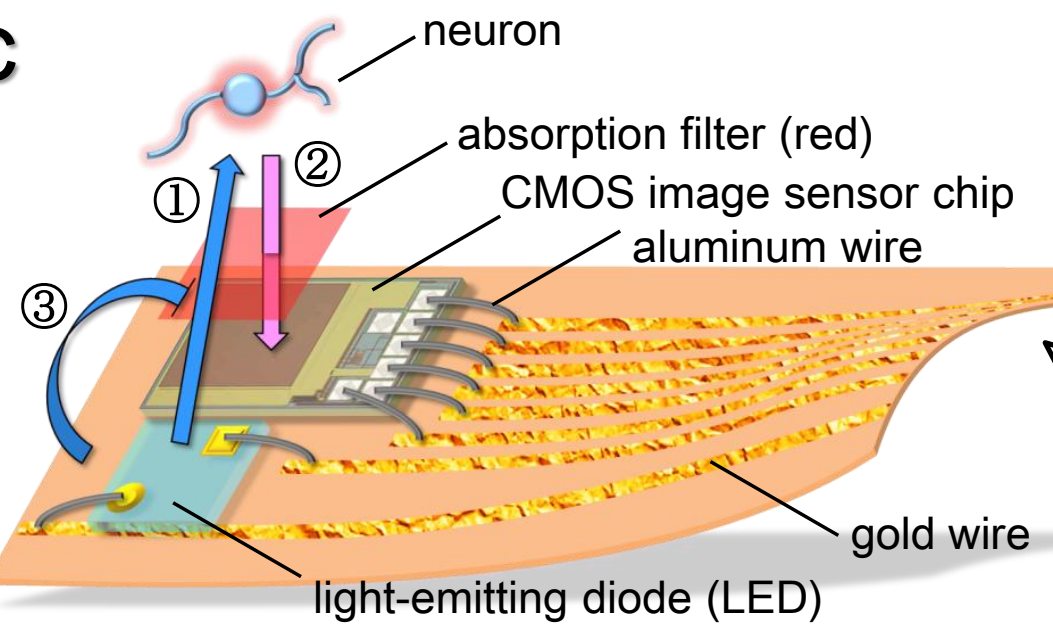

flexible polyimide substrate

D

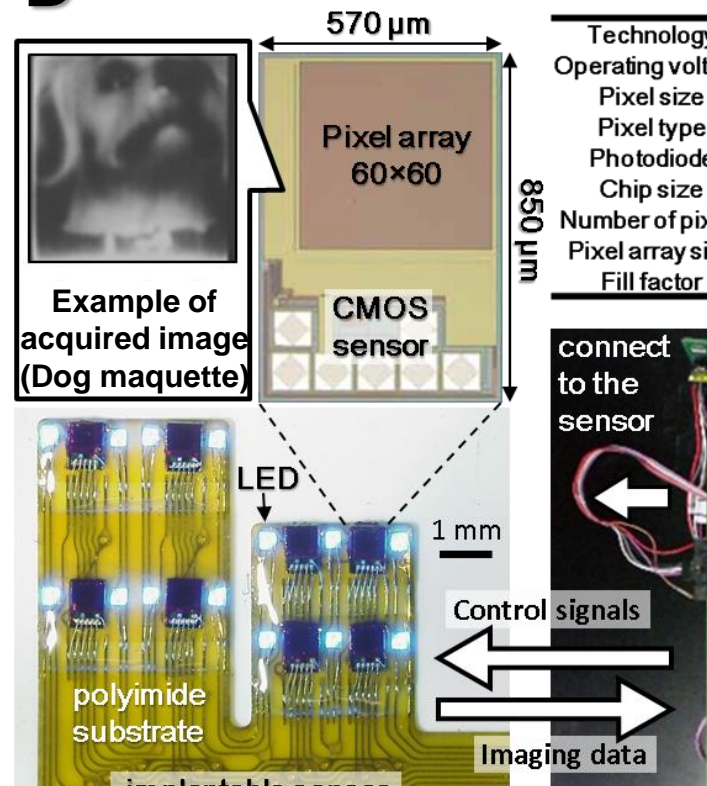

MOS $(2-p o l y ~$
$3.3 \mathrm{~V}$

$7.5 \mu \mathrm{m} \times 7.5 \mu \mathrm{m}$ Three transistor APS $\mathrm{N}$-well / P-substrate junction $570 \mu \mathrm{m} \times 850 \mu \mathrm{m}$ $60 \times 60$ $450 \mu \mathrm{m} \times 450 \mu \mathrm{m}$ $35 \%$

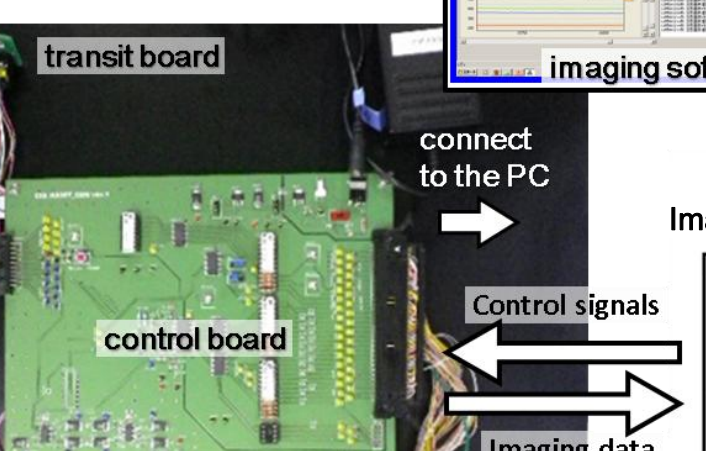

Imaging data
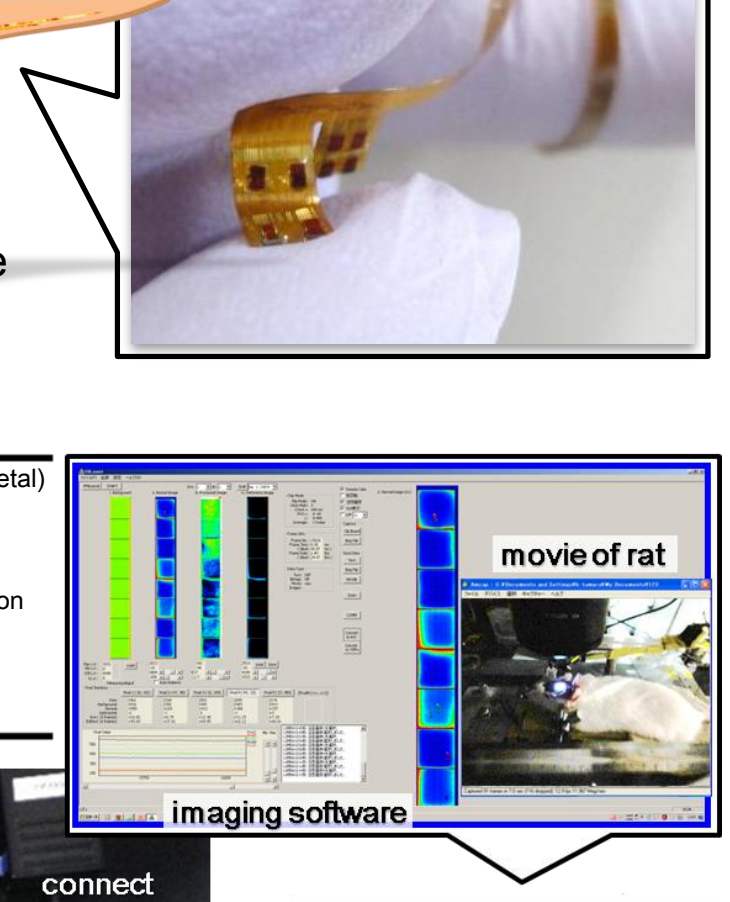

Controlling device/ Imaging/Recording on one PC

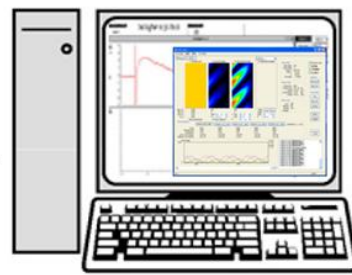


Fig. 2
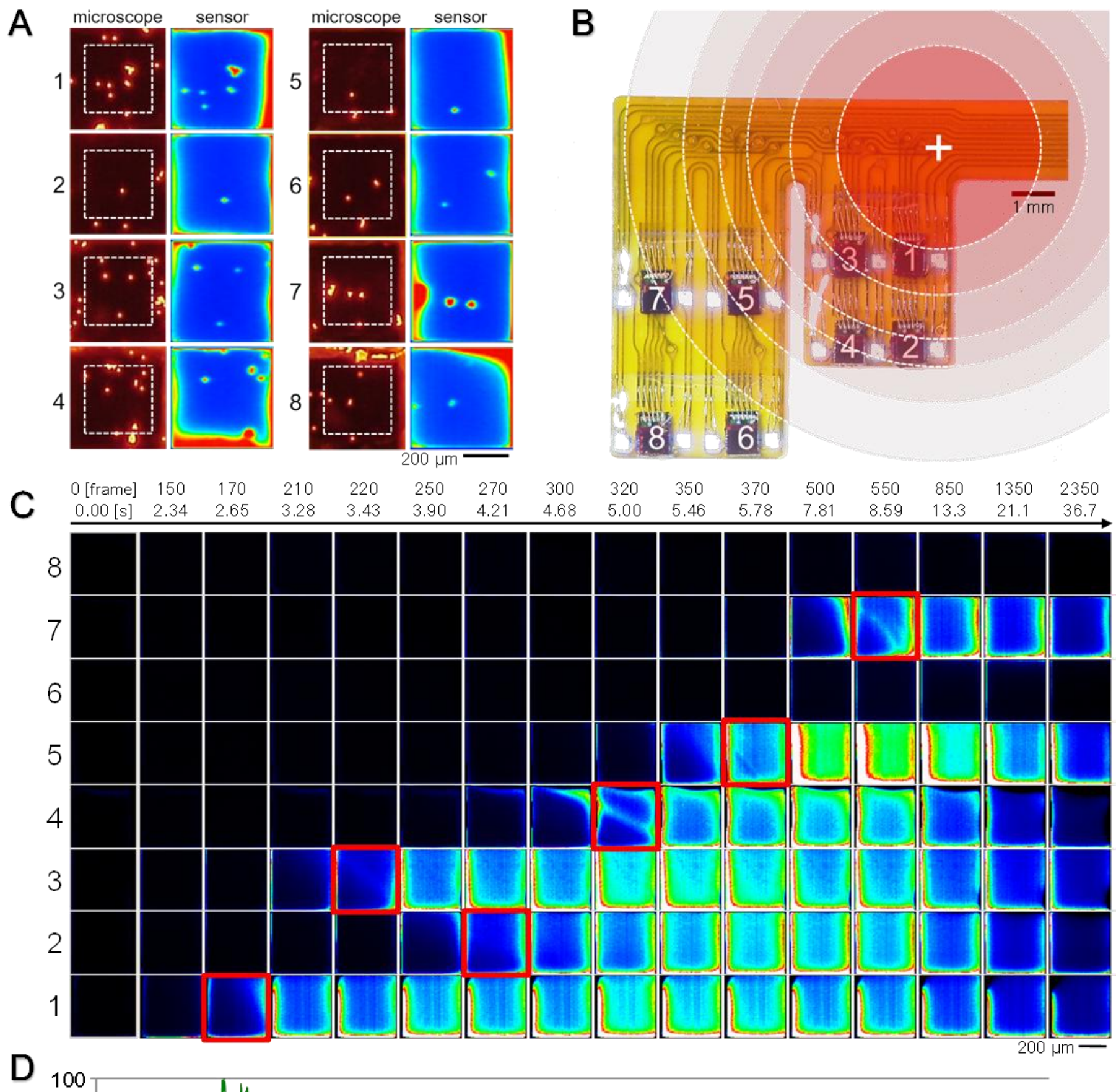

D

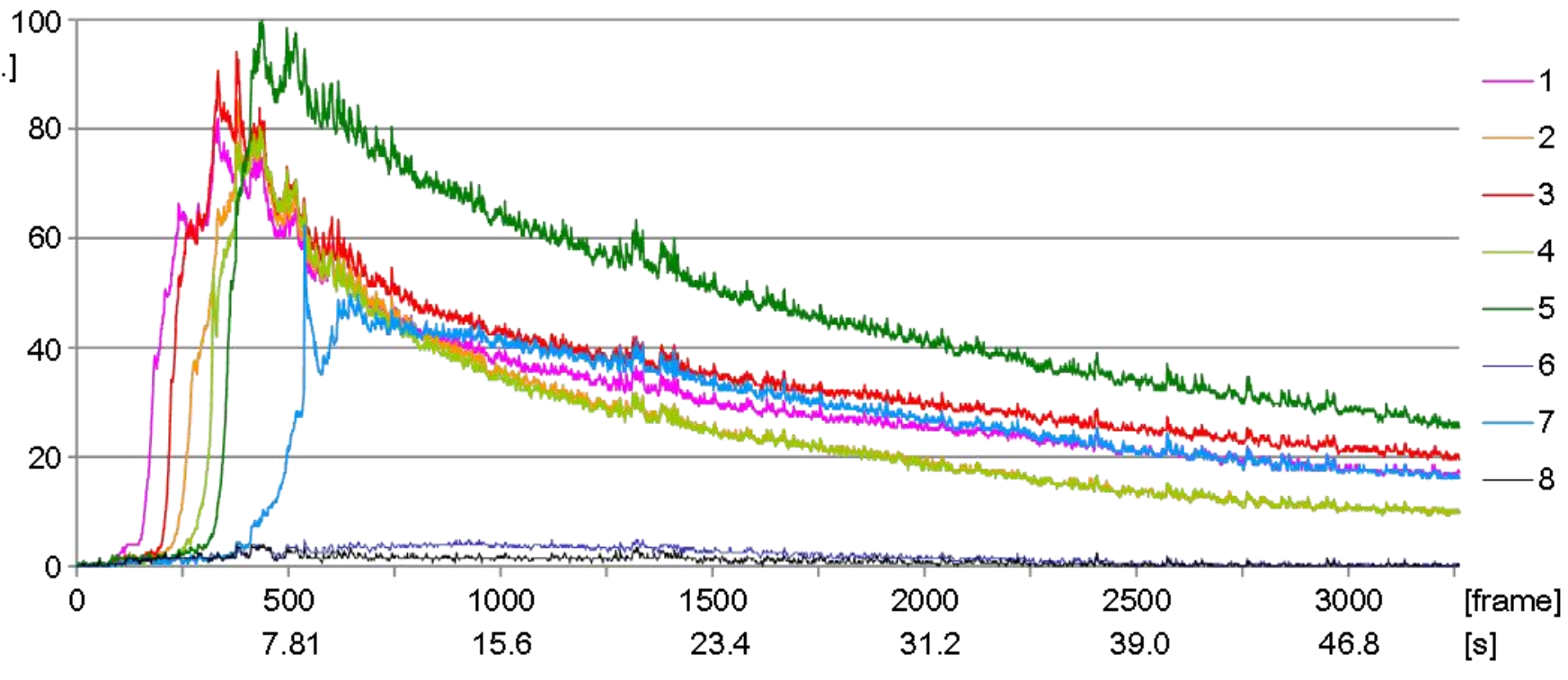




\section{Fig.3}

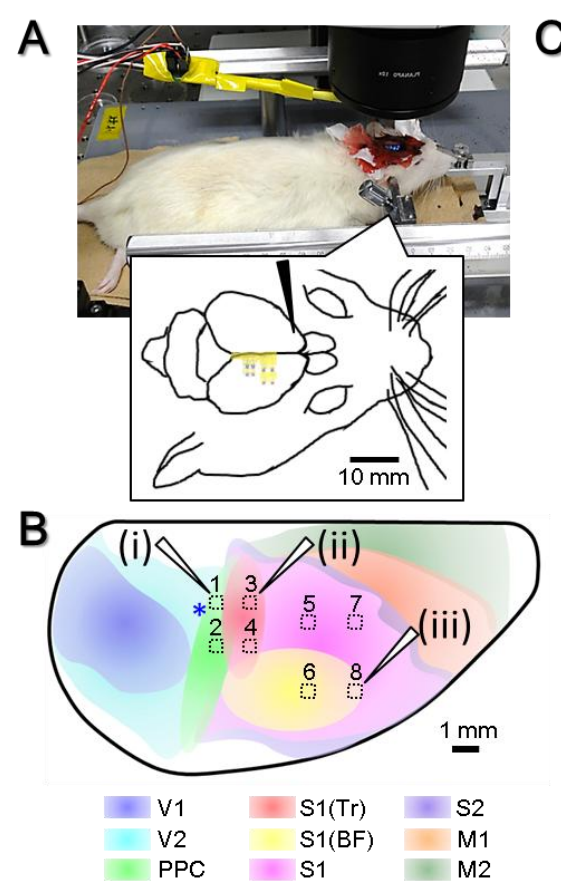

D $\mathrm{C}-1$

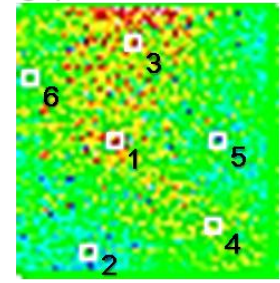

F (i)

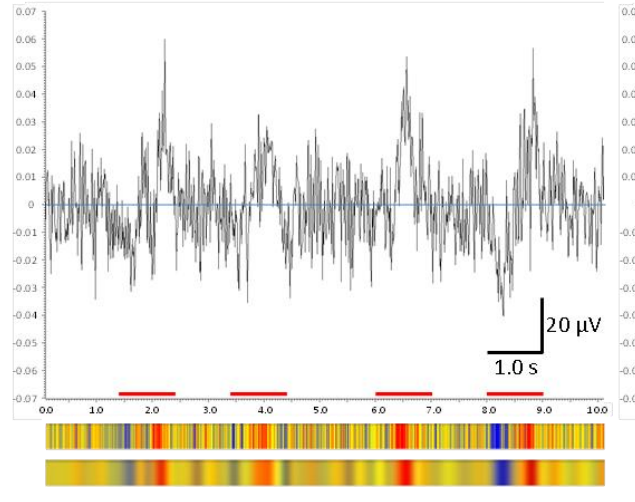

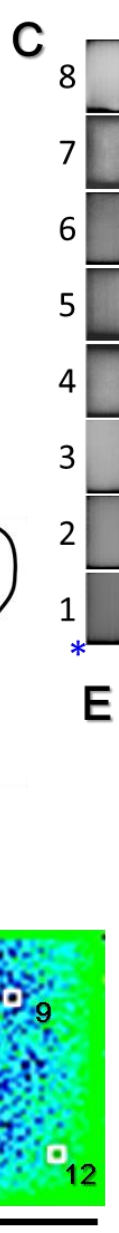

(ii)
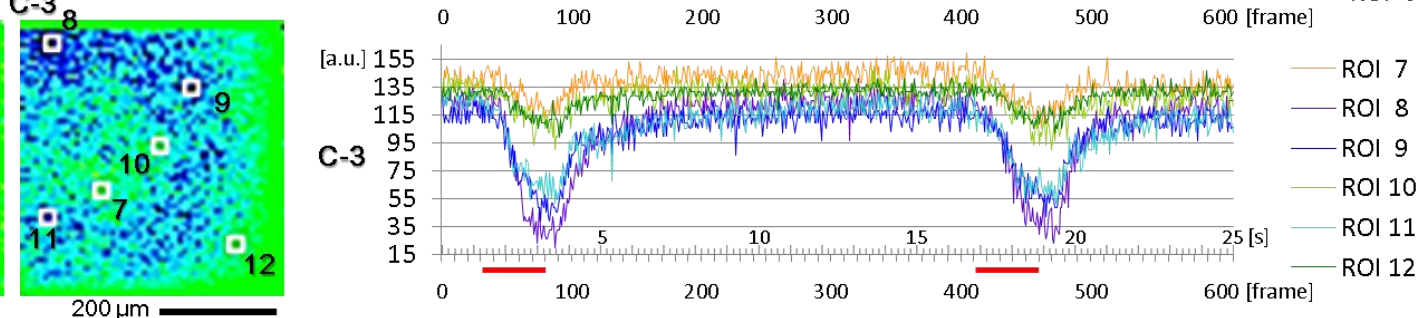

(iii)

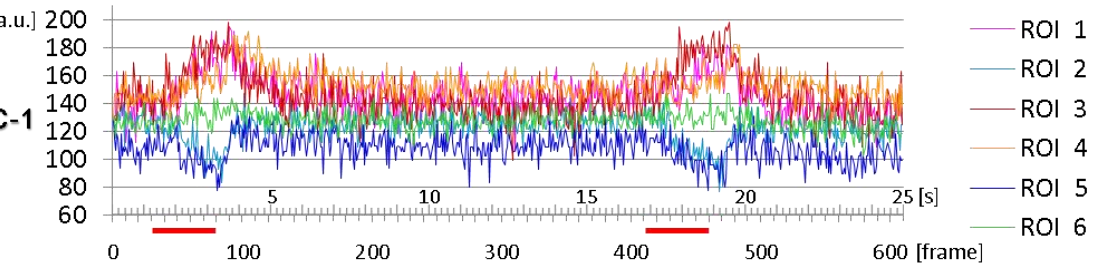

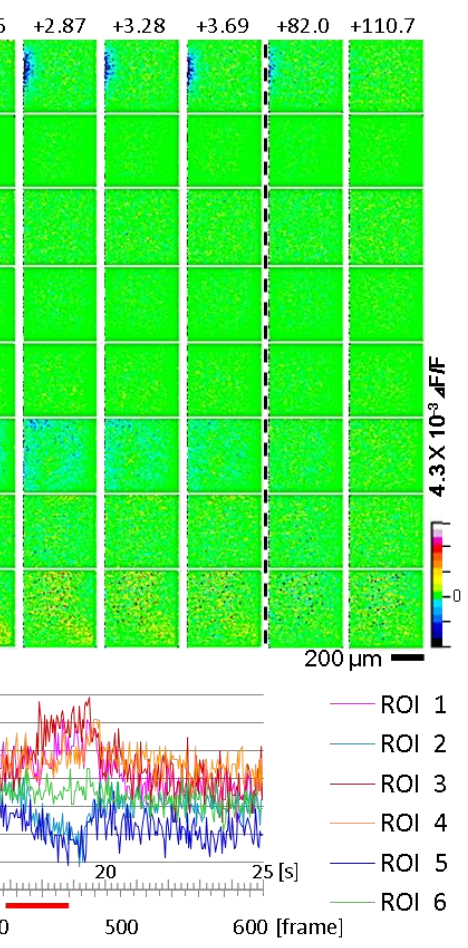
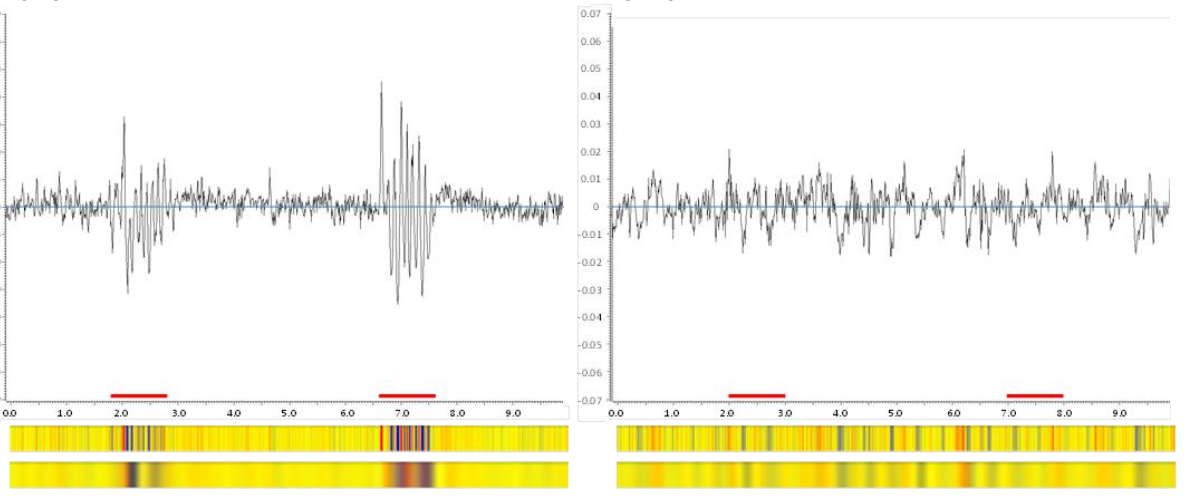